\title{
Generalized dominant dystrophic epidermolysis bullosa
}

INSERM

\section{Source}

INSERM. (1999). Orphanet: an online rare disease and orphan drug data base.

Generalized dominant dystrophic epidermolysis bullosa. ORPHA:231568

Generalized dominant dystrophic epidermolysis bullosa (DDEB-gen) is a subtype of dystrophic epidermolysis bullosa (DEB, see this term), formerly known as DDEB, Pasini and Cockayne-T ouraine types, characterized by generalized blistering, milia formation, atrophic scarring, and dystrophic nails. 